\title{
Application of "Cross-border Design" Concept in Industrial Product Design
}

\author{
Xing Yadan \\ Guangzhou Huali Science and Technology Vocational College, Guangzhou, China, 511325
}

Keywords: cross-border design; industrial product design; interior design; innovation

Abstract: The continuous advancement of the modern society has promoted the continuous improvement of industrial theory. Every day, various new ideas and thoughts are emerging. These changes are enriching and facilitating people's lives. In this era, the concept of cross-border design came into being. This mode of thinking first integrated the innovations of various design elements, and then created on the basis of industrial products. This paper focuses on the industrial product design and the interior product design, analyzes the differences between them, and concludes that there is some connection between them. The conclusion that "crossover" is feasible can be fully used for reference to the inspiration and design ideas of interior design.

\section{Introduction}

The idea of cross-border design represents a new type of life attitude and aesthetic experience for modern people. It gives new charm to industrial products and makes them more attractive to people. From the English definition of cross-border design, it can be seen that it represents the interaction and cooperation between various industries. For now, the idea of cross-border design has already triggered an uproar in the design world and has become a popular design.

With the constant impact of the industry 4.0 wave, China's manufacturing industry is also in an important transition period. In response to the increasingly serious homogenization phenomenon in the industrial product market, enterprises pay more attention to improving R\&D capabilities and exploring core technologies, and also put forward more requirements for product design. Industrial product design also requires a diverse approach to design and design strategies to provide a more diverse range of products and services to businesses and society ${ }^{[1]}$.

\section{Design "cross-border"}

With the advent of the "Internet +" era, "cross-border" runs through various industry sectors. The field of design is no exception, and cross-border cooperation in many fields brings new vitality to the design community. In recent years, designers have become more and more keen on crossing the boundaries of disciplines and industries, breaking through the boundaries of the field, and carrying out design innovation ${ }^{[2]}$.

In fact, in the field of design, "cross-border" is a very common phenomenon. The origins of "cross-border design" can be traced back to the beginning of design. It can be said that there is no 
time to cross the border, nowhere to cross. The cross-border between the many sub-disciplines that occur in the design discipline is also more frequent and natural, because these sub-disciplines, although different in name, follow the same design rules, which provide natural conditions for the "cross-border" between them.

With the rapid increase in productivity, the homogenization of the industrial product market is becoming more and more serious. "Closed-door construction" will only create obstacles for the design of industrial products and the development of the industry. Industrial product design can add more possibilities through "cross-border". By finding intersections in different product designs, designers can provide more design materials, expand designers' thinking and broaden designers' horizons. On the other hand, for users, "cross-border" can bring a new visual and psychological experience. In the face of the diversified needs of the market, industrial product design also needs to draw resources and inspire from other fields to provide conditions for design innovation ${ }^{[3]}$.

\section{Impact analysis of cross-border design}

As a new type of design concept, cross-border design has attracted the attention of many people, but designers must also rationally look at cross-border design and grasp the design direction. The result of cross-border design is that both failure and success coexist. This paper analyzes both positive and negative aspects.

\subsection{Positive influence}

(1) Increased possibilities for industrial product design

Cross-border design has good permeability characteristics, finding intersections in different product designs, and providing possibilities for many designs that were previously unimaginable. On the one hand, cross-border design provides designers with a wealth of design materials to broaden their horizons; on the other hand, cross-border design expands the communication surface, stimulates more creative sparks, and promotes the progress of the design industry.

(2) Bring new experiences to users

Cross-border design products combine a variety of new technologies, fashion elements and manufacturing techniques to bring new visual and psychological experiences to users. Visually, the unique shape of the cross-border design product is both new and exciting. An alternative design concept can give those who like novelty full satisfaction. In addition, cross-border design products often have multiple functions, users are not only easy to use, but also can reduce the cost of repeated purchases.

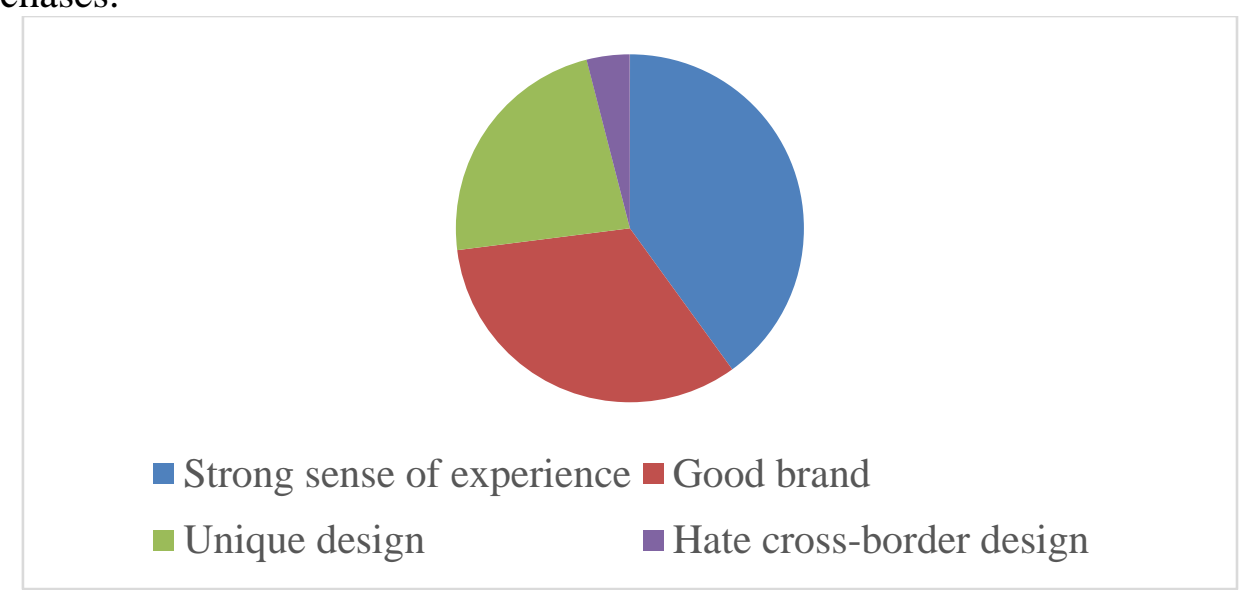

Figure 1 User's favorite reasons for using industrial products manufactured by cross-border design 
(3) Achievement brand strategy

Cross-border design can enrich the brand's inner cultural connotation, enhance brand value, and strengthen the brand's social influence. Major brand companies share customer resources through cross-border cooperation, which is conducive to achieving greater economic benefits. Figure 1 is an impact questionnaire on cross-border design ${ }^{[4]}$.

\subsection{Negative impact}

Cross-border design can bring new user experience and create new value, but if you do not grasp the scale, lack of careful planning and development vision, it will have a great negative impact. The problems faced by cross-border design will cause the company to be in trouble if it is not properly handled.

\section{Industrial product design and interior design}

\subsection{Industrial product design is closely linked to interior design}

There are many subjects in the design process, and the industrial design also covers a wide range, including not only design management, but also product design and environmental design. It must be clarified here that industrial products are included in the design of the product, while interior design is part of the environmental design. It can be seen that both of these are under product design, and the connection is especially close. At the designer's level, many industry giants can not only serve as product designers, but also competent environmental designers. Among them, Mies van der Rohe is a typical representative ${ }^{[5]}$.

\subsection{Comparison of the development of interior design and industrial product design in China}

In recent years, with the continuous improvement of people's living standards, interior design has been greatly developed, and in the process of development, Chinese traditional elements have been incorporated, forming a Chinese style design style. Moreover, according to a large number of market research, Chinese-style design has great market potential, especially for the home improvement market, which has a huge market share. In contrast, today's market is basically "European", "American" and so on, the local manufacturers lack the spirit of innovation, just blindly copying. This is very unfavorable for the future of local manufacturing and must be fundamentally changed. After analysis, it can be seen that the Chinese style interior design is to some extent utilized the architectural culture foundation of China for thousands of years, but in contrast to industrial product design, it seems that time is more rushed and experience is even more lacking.

\subsection{Cross-border of interior design and industrial product design}

In essence, industrial product design and interior design are inextricably linked. If you can use the cross-border, refine the ideas, design essentials and design elements of interior design and apply them to industrial product design. A new industrial product design. This is conducive to the appearance of product innovation, and also has a certain role in promoting the design of Chinese style industrial products. As shown in Figure 2, through the cross-border, the shape of the closed-packing robot not only satisfies the psychological needs of consumers, but also has obvious regional recognition, which can quickly associate with Chinese culture. 


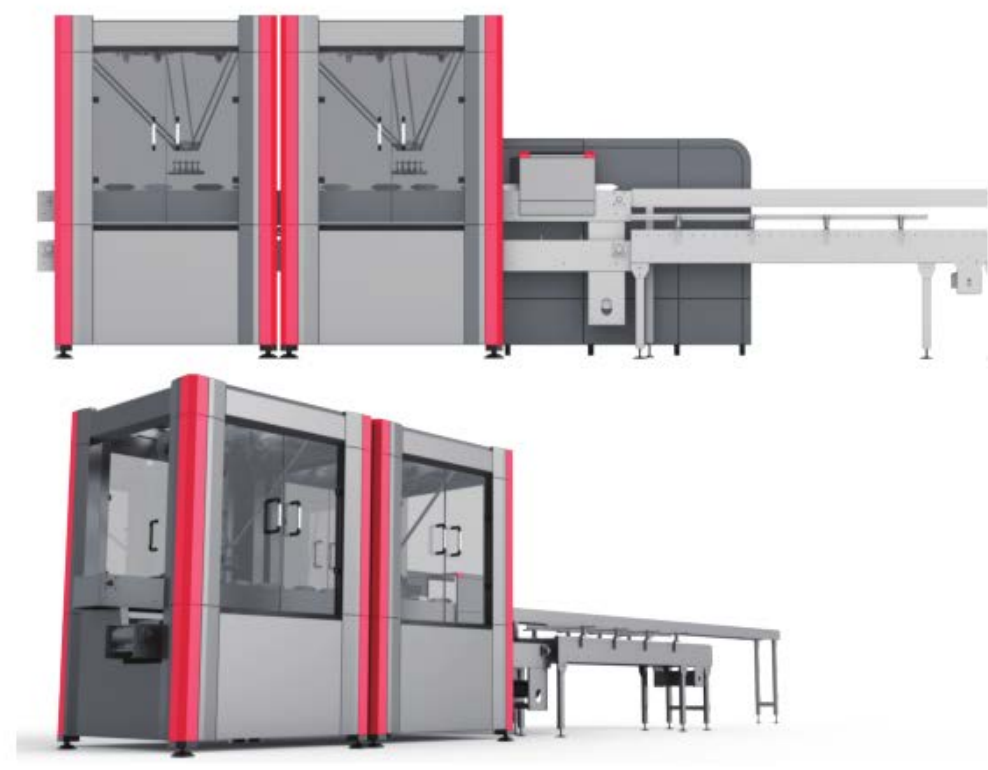

Figure 2 Closed boxing robot program renderings

On the quantitative level, there are many factors involved in interior design. The introduction of “cross-border" ideas can apply many valuable interior design experiences to industrial product design.

On the quality level, the market has the function of screening. Those designs that meet the aesthetic needs of the contemporary people will be preserved. On the contrary, those who violate the aesthetic concept will be eliminated by the market, which is determined by the attributes of the market. Therefore, from this point of view, the current interior design elements are in line with people's requirements for beauty. After that, after scientific transformation, these excellent design elements can be better applied to industrial design.

\section{Conclusions}

Cross-border design advocates breaking industry barriers and designing more time-oriented products by changing mindsets, which will be the development direction of industrial product design in the future. Through the cross-border design, the combination of industrial product design and interior design can make use of the development advantages of interior design to make up for the deficiencies of industrial product design, which can provide a new design method and design strategy for industrial product design. The vast number of industrial product designers need to understand the development trend of the society and the value orientation of people, integrate the artistic essence of each discipline, and create more novel products.

\section{References}

[1] Liu Kun. Application of the concept of "cross-border design" in industrial product design [J]. Tomorrow's fashion, 2018(9).

[2] Fan Yipeng, Chen Yujing. Application of "cross-border design" concept in industrial product design[J]. China Packaging, 2017, 37(6): 27-30.

[3] Han Xiong. On Cross-border Design in Industrial Design Teaching[J]. Science and Education Journal, 2012(13): 54-54.

[4] Zhou Rui. Research on Cross-border Trends in Current Industrial Design[J]. Journal of Nanjing University of the Arts: Art and Design, 2008(3): 193-196.

[5]Zhou Wei, Huang Shengbin. "Boundary" and "Transboundary" in Design[J]. Creative \& Design, 2010(1): 31-32. 